\title{
Physical quantities useful for quality control of quantitative SPECT/CT imaging
}

\author{
Sara Kurkowska®, Bozena Birkenfeld $\odot$, Hanna Piwowarska-Bilska® \\ Department of Nuclear Medicine, Pomeranian Medical University, Szczecin, Poland
}

[Received 18 I 2021; Accepted 04 VI 2021]

\begin{abstract}
SPECT/CT imaging is transitioning from solely qualitative applications to quantitative analysis. Quantitative SPECT/CT systems require proper calibration, optimization and quality control. Various types of modern SPECT/CT scanners have different software for calibration and quality control (QC). There is still no standardization in this regard for quantitative SPECT/CT. This issue hinders the exchange of obtained results across centers and stunts the development of repeatable and reproducible measurements. The unification and standardization of calibration and quality control techniques for quantitative SPECT/CT systems is currently a pressing need for nuclear medicine departments.

The present study presents three selected physical quantities characterizing the quality of quantitative SPECT/CT system and seven quantities, currently used in the literature, to assess the quality of quantitative SPECT/CT images. The measurement of these parameters requires the use of standard gamma camera software for $\mathrm{QC}$, external programs for quantitative analysis of recorded data and clinical software. The authors hope this will help physicists who are willing to perform quantitative SPECT/CT in their departments.
\end{abstract}

KEY words: SPECT/CT; quantitative SPECT/CT; SUV

Nucl Med Rev 2021; 24, 2: 93-98

\section{Introduction}

During the last decades, nuclear medicine is undergoing a significant change, emphasizing the quantitative quality of single photon emission computed tomography (SPECT) images. The introduction of hybrid devices, combining SPECT gamma cameras with a computed tomography (CT) scanner, enabled quantitative evaluation of images thanks to CT-based attenuation correction. New techniques of images reconstruction, based on iterative methods, reduced errors in absolute quantification in SPECT thanks to scatter and attenuation corrections. This permitted the introduction of quantification of radiopharmaceuticals uptake in healthy and pathological tissues and it is commonly expressed as standardized uptake value (SUV). Moreover, quantitative SPECT/CT images play a crucial role in internal dosimetry used for individualized radionuclide treatment planning.

Correspondence to: Sara Kurkowska

Department of Nuclear Medicine, Pomeranian Medical University, Unii

Lubelskiej 1, 71-344 Szczecin, Poland

e-mail: sarakurkowska95@gmail.com
For positron emission tomography (PET)/CT systems, the National Electrical Manufacturers Association (NEMA) has defined the standards which allow performing the quality control of quantitative images [1]. Such standards, though, are not defined for quantitative SPECT/CT imaging, yet. This issue hinders the exchange of obtained results across centres and stunts the development of repeatable and reproducible measurements.

While waiting for a set of standard procedures, active research is conducted to evaluate the accuracy and precision of quantitation [2-4]. To verify the quality of quantitative images obtained with SPECT/CT, many authors try to adopt the methods employed in PET or, if necessary, modify them as needed. According to Dickson et al. [5], now is the time to work together as a community to make this potential a reality.

The present study reviews physical quantities, currently used in literature, to assess the quality of quantitative SPECT/CT images. The authors hope this will help physicists who are willing to perform quantitative SPECT/CT in their departments.

\section{Basic requirements for obtaining quantitative SPECT/CT images}

The basic requirement for obtaining reliable results of quantitative image analysis is to perform acquisitions with SPECT/CT

This article is available in open access under Creative Common Attribution-Non-Commercial-No Derivatives 4.0 International (CC BY-NC-ND 4.0) license, allowing to download articles and share them with others as long as they credit the authors and the publisher, but without permission to change them in any way or use them commercially. 
systems, which have the correct results of periodic quality control and calibration tests recommended by the manufacturer and compliant with NEMA guidelines. Standard instrument calibrations and quality control tests (including tests of uniformity, the centre of rotation, SPECT/CT spatial co-registration, as well as CT system tests) should be performed regularly, following the schedule recommended by producers. The clinical use of gamma cameras that do not show the stability of the measured parameters forces users to test and calibrate the systems more often.

Quantitative image reconstructions must be performed by iterative methods such as maximum likelihood expectation maximization or ordered subsets expectation maximization (OSEM), because only these reconstruction methods allow to perform attenuation and scatter corrections. The exact correction for attenuation is performed based on the attenuation maps, calculated from the transmission images of a patient, obtained with the CT component of the SPECT/CT system during one hybrid imaging session. Low-dose CT acquisitions, used to ensure radiological protection of the patient, are sufficient to correct the attenuation in SPECT images. The attenuation corrections are introduced at the stage of reconstruction of the scintigraphic slices.

A popular and relatively accurate method of scattered photon correction is acquisition with the triple energy window [6]. It allows evaluating the number of scattered photons based on the measurement of the counts in the projections collected in two additional energy windows, placed on both sides of the photopic. In the software of modern gamma cameras, the scattering correction is built directly into the reconstruction algorithm.

The correction of the gamma camera's resolution recovery is not necessary, because it does not affect the accuracy of the quantitative measurement of the activity distribution. Nevertheless, it is recommended since improving the resolution of the images facilitates the segmentation process.

\section{Selected quantities characterizing the quality of quantitative SPECT/CT system}

The physical quantities described in this work are the quantities most frequently reported in the literature that characterize the quantitative SPECT/CT quality.

\section{Sensitivity}

Each manufacturer of gamma cameras recommends a specified, repeatable technique for measuring the sensitivity of the gamma cameras. For example, Siemens, before the quantitative acquisition of scintigraphic images, requires a one-time volume sensitivity measurement using a cylindrical phantom filled with a homogeneous radioisotope solution. Afterwards, the monthly planar measurement of the sensitivity of the point source in a dedicated test tube should be performed. GE Healthcare recommends planar measurement of the detectors' sensitivity using a flat plastic dish (e.g., standard Petri dish), containing a homogeneous radioisotope solution. Commercial gamma camera software, within the set of programs for quality control, contains dedicated applications for measuring the sensitivity of the system. To compare the sensitivity of different types of gamma cameras, one should follow the recommendations of NEMA 1-2018 [7] regarding the measurements of planar sensitivity test of detectors and volume sensitivity test of SPECT system.

\section{Cross-calibration}

To enable quantitative measurements, all SPECT images reconstructed in counts per pixel must be converted to the activity concentration $(\mathrm{Bq} / \mathrm{mL})$ units. In this way, it is possible to analyse the data by applying the SUV. There are two ways to convert it.

Firstly, the reconstructed counts in the SPECT volume can be directly related to the acquired counts in the projection data. In this situation, calibration is related to the absolute value of the camera sensitivity from the planar measurement, in units of counts per second per $\mathrm{kBq}(\mathrm{cps} / \mathrm{kBq})$.

Another method uses the cross-calibration factor (CCF), which is determined from a reconstructed image, relating the counts to the radioactivity concentration. The CCF can be evaluated using a cylindrical homogeneous phantom and is defined by

$$
\operatorname{CCF}\left[\frac{c p s / m l}{B q / m l}\right]=\frac{\frac{\mu}{t \times n \times v}}{A_{c}},
$$

where $\mu$ is the total counts in VOI in the reconstructed image, $t$ is the time per projection, $n$ is the number of projections, $v$ is the VOI volume, $A_{c}$ and is the true activity concentration in the phantom.

In the Q.Dose software [8], the calibration is performed using the Scaling Factor, which is the reciprocal of the CCF

$$
S F\left[\frac{B q / m l}{c p s / m l}\right]=\frac{A_{c}}{\frac{\mu}{t \times n \times v}} .
$$

\section{Spect reconstructed spatial resolution}

An important quantity to assess the quality of quantitative tomographic imaging is the reconstructed spatial resolution, measured in air and a dispersion medium. The reconstructed spatial resolution of the SPECT system is defined as the full-width at half-maximum (FWHM) of the line spread function (LSF) or of the point spread function (PSF) with an imaging collimator installed. This measurement should be completed with the full width to the tenth of the maximum because the PSF or LSF may deviate from the Gaussian distribution. NEMA [7] recommends the use of 3 capillary tubes (e.g., syringe needles) with an inner diameter $1 \mathrm{~mm}$ as radioactive sources. They should be positioned parallel to the principal orthogonal axes of the camera to avoid broadening the LSF. Measuring the Reconstructed Spatial Resolution with Scatter requires placing 3 capillary tubes in a cylinder of water. Following NEMA guidelines, cross-sections through source centres (slices of thickness $10 \pm 3 \mathrm{~mm}$ ) should be reconstructed using the filtered back-projection technique with the ramp filter.

\section{Dead-time}

Imaging studies of patients who have just received radionuclide therapy, where relatively high activities of radionuclides are usually administered, may be affected by considerable dead time (DT) effects. These are caused mainly by the pile-up in the gamma camera detector and electronics. Therefore, for any post-therapy imaging study where quantitative information is required (e.g., for image-based dosimetry calculations, tumour staging and evaluation of therapy outcomes), DT corrections should be performed. 
Ryu et al. [4] measured the DT effect of the SPECT system using a cylindrical phantom with high initial activity for [99mTC] and ${ }^{177} \mathrm{Lu}$. The DT constants were estimated based on the concepts of paralyzable $\left(\tau_{p}\right)$ and non-paralyzable $\left(\tau_{N P}\right)$ DTs and the corresponding two-component model introduced by Cranley et al. [9]. The DTs in the two-component model can be easily determined through a least-squares fitting for the decaying source method. To evaluate the paralyzable and non-paralyzable components of the system DT, the authors fitted eq. (3) to the observed data,

$$
r^{\prime}=\frac{r}{e^{r \tau_{P}}+\left(\frac{\tau_{N P}}{\tau p}-1\right) r \tau_{P}},
$$

where $r$ ' is the observed count rate, $r$ is the true count rate, $\tau_{p}$ is the paralyzable and $\tau_{N P}$ the non-paralyzable constant. The fitting was stopped when $\mathrm{R}^{2}$-value was equal to 1.0 .

\section{Quantities describing the quality of the quantitative SPECT/CT image}

\section{Accuracy of activity recovery}

The accuracy of activity recovery is simply the ratio between the activity concentration, measured in the reconstructed SPECT image $\left(a_{c}\right)$, and the true activity concentration $\left(A_{c}\right)$, measured during phantom preparation. This quantity can be measured for the cylindrical homogeneous and the anthropomorphic phantoms. Depending on the chosen phantom, the regions of interest (ROIs) can be defined in the background or in the spheres, when an anthropomorphic phantom, for example, NEMA/International Electrotechnical Commission (IEC) NU2 phantom, is used.

The background calibration factor (Bg.cal) is defined as

$$
\text { Bg.cal }=\frac{a_{c, b g}}{A_{c, b g}}
$$

where $a_{c, b g}$ is the activity concentration measured in the reconstructed SPECT phantom background and $A_{c, b g}$ is true activity concentration inside the phantom. Gnesin et al. [2] determined $a_{c, b g}$ as the average on 5 circular ROls of $16 \mathrm{~cm}$ diameter centred on the cylinder axis, placed at different axial locations as in Figure 1.

For spheres, recovery coefficients (RCs) are defined as

$$
\begin{aligned}
& R C_{j, \max }=\frac{a_{c_{s p h} j, \max }}{A_{c, s p h}}, \\
& R C_{j, A 50}=\frac{a_{c_{s p h} j, A 50}}{A_{c, s p h}},
\end{aligned}
$$

where $j$ is the number of the sphere, $a_{c_{\text {sph }} \text {, max }}$ is the measured maximum voxel value (in terms of activity concentration) for a given spherical insert, $A_{c, s p h}$ is the true activity concentration in the sphere, $a_{c_{\text {sph }} \text {,A50 }}$ is the average voxel value for each hot insert VOI defined by a 3D isocontour at 50\% adapted for background [10]

In their study, Peters et al. [11] determined the RC for each sphere performing five measurements and, then, they evaluate the repeatability of these measurements using the median absolute deviation (MAD) for each sphere diameter according to

$$
M A D=\operatorname{median}\left(\left|R C_{i}-R C\right|\right),
$$

where $R C_{i}$ is the recovery coefficient of measurement $i$ and $R C$ is the median recovery coefficient of all five measurements.

The white paper published by GE Healthcare [12] shows a modified equation for the accuracy,

$$
\operatorname{Accuracy}(\%)=\left(1-\frac{\left|A_{c, s p h, j}-a_{c, s p h, j}\right|}{A_{c, s p h, j}}\right) \times 100 .
$$

\section{Image noise}

The coefficient of variation (COV) is a parameter used to evaluate image noise. It is expressed as the ratio between the standard deviation $\left(\sigma_{b g}\right)$ and the mean value of activity concentration measured in the phantom background $\left(\bar{a}_{c, b g}\right)$,

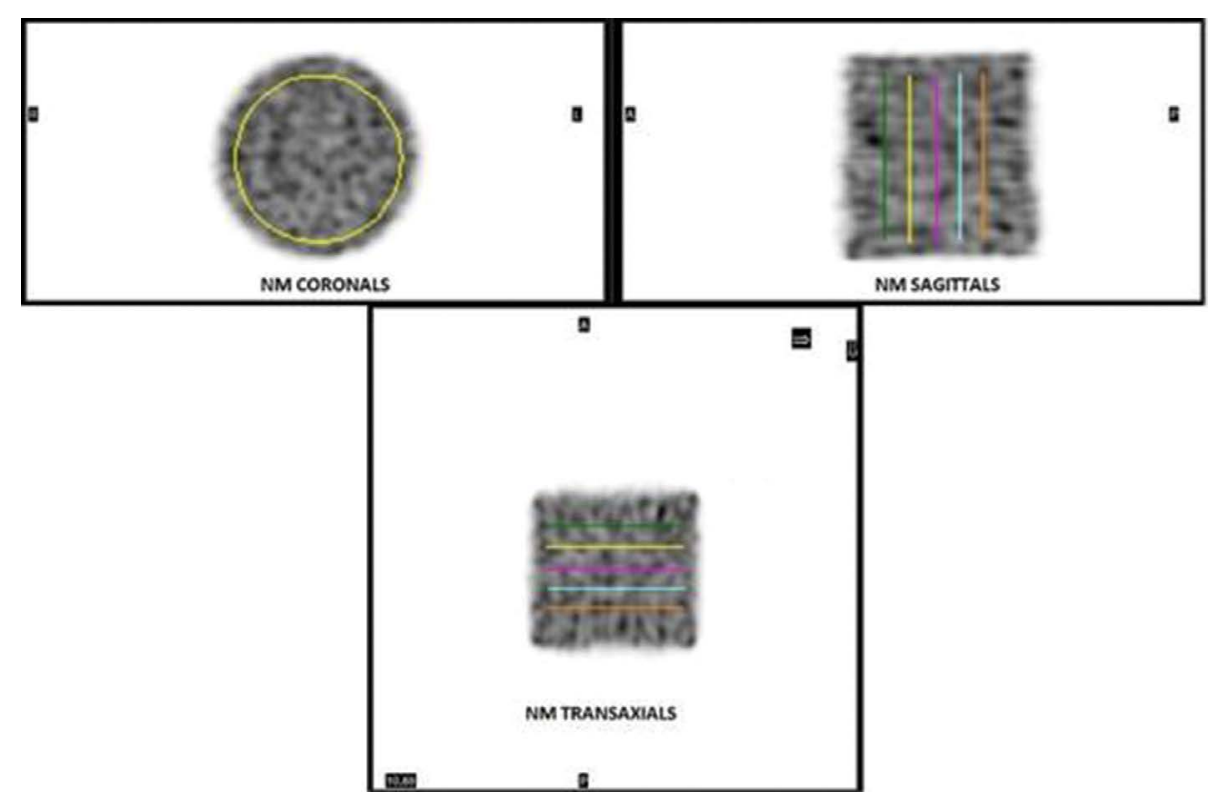

Figure 1. Reconstructed image of Phantom Well Counter Calibration Uniform Cylindrical performed in Q.Metrix software from GE Healthcare. 5 circular ROls of $16 \mathrm{~cm}$ diameter centred on the cylinder axis were placed at different axial locations and used to calculate Bg.cal 


$$
\operatorname{COV}(\%)=\frac{\sigma_{b g}}{\bar{a}_{c, b g}} \times 100
$$

The background mean value of activity concentration is determined as the mean value of several different ROls and $\sigma_{b g}$ is the standard deviation of the mean of these ROls.

Koopman et al. [13] in placed three rectangular ROls within the phantom background in three axial planes, obtaining nine ROls. For each ROI, the image COV was determined using eq. (9). The COV for a reconstructed image was obtained by taking the average of the nine measured COVs. The technique of delineating ROls is presented in Figure 2.

This quantity can be measure for the cylindrical homogeneous phantom and the NEMA/IEC NU2 phantom.

\section{Total activity deviation}

The total activity deviation is defined by

$$
\Delta A_{\text {tot }}(\%)=\frac{A_{\text {tot }, \text { rec }}-A_{\text {tot }}}{A_{\text {tot }}} \times 100
$$

where $A_{\text {tot, rec }}$ is the total activity in the phantom measured in the reconstructed image, and $A_{\text {tot }}$ is the true total activity of radionuclide measured during phantom preparation.

This quantity can be measured for the cylindrical homogeneous phantom and the NEMA/IEC NU2 phantom.

\section{Contrast recovery}

To evaluate the contrast recovery, the NEMA/IEC NU2 phantom with hot spheres should be used. The hot contrast for each hot sphere is calculated by

$$
Q_{H, j}(\%)=\frac{\left(a_{c, s p h, j} / a_{c, b g}\right)-1}{\left(A_{c, s p h, j} / A_{c, b g}\right)-1} \times 100 .
$$

The ideal ratio is equal to $100 \%$.

\section{Residual error in scatter and attenuation corrections}

The relative error in the lung, $\Delta C_{\text {lung, }}$, allows evaluating the residual error in scatter and attenuation corrections using an anthronomornhic, nhantom with lund insert and it is defined as

$$
\Delta C_{\text {lung }}(\%)=\frac{a_{c, l u n g}}{A_{c, b g}} \times 100,
$$

where $a_{c, \text { lung }}$ is the average activity concentration measured in a cylindrical VOI, with a $3 \mathrm{~cm}$ diameter and a length of $16 \mathrm{~cm}$, placed into the lung insert [2].

\section{Discussion}

Quantitative analysis of SPECT/CT images is a difficult task. Many physical effects hinder proper quantification. Among the most important ones are blurring introduced by the collimator response function, the limited spatial resolution and associated partial volume effect, photon attenuation and the contribution in the images of events arising from photons scattered in the objects. There are, however, algorithms and methods used to compensate for these effects.

Despite these limitations, SPECT/CT is transitioning from solely qualitative applications to quantitative analysis.

Seret et al. [14] investigated four SPECT/ CT systems focusing on their quantitative capabilities. They found that, in objects whose dimensions exceeded the SPECT spatial resolution by several times, quantification seemed to be feasible within $10 \%$ error limits. A partial-volume correction strategy remains necessary for smaller structures. In a study performed by Zeintl et al. [15], they report an average quantitative accuracy within $3.6 \%$ in phantoms with different-sized spheres and $1.1 \%$ in patients with a focus on the bladder when using [ $\left.{ }^{99 m} \mathrm{Tc}\right]$.

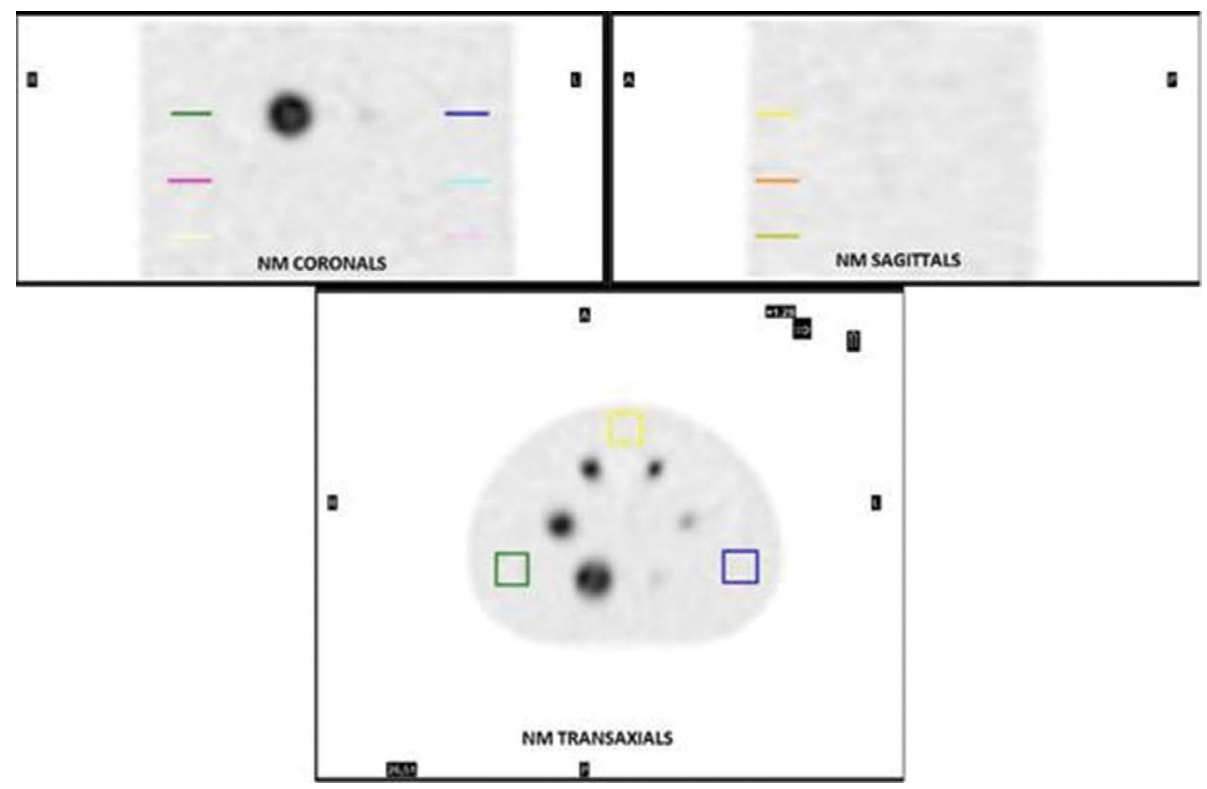

Figure 2. Reconstructed image of NEMA Body Phantom/IEC NU2 performed in Q.Metrix software from GE Healthcare. Three rectangular ROls in three different axial planes within the phantom background were used to calculate COV. COV — coefficient of variation; IEC - International Electrotechnical Commission; NEMA — National Electrical Manufacturers Association 
Dead-time is an important effect in quantitative SPECT. In terms of radionuclide therapy, where injected activities are very high, dead-time losses can be substantial and cause an underestimation of the doses received by organs. It is essential to correct for this effect during dosimetric studies. Dead-time correction is particularly important for radionuclides with multiple photon emissions such as ${ }^{131}$, as photons not included in the energy window also contribute to dead time. In a study on the dosimetric impact of dead-time correction after a $4 \mathrm{GBq}$ therapeutic injection of ${ }^{131}$, correction for count losses led to an $11 \%$ increase in whole-body time-integrated activity [16]. For descriptions of practical methods for calculating corrections for DT effect for quantitative studies using high ${ }^{177} \mathrm{Lu}$ activities, see articles [17, 18].

In a study from 2012, Dewaraja et al. [19] showed that a planar measurement of a point source is sufficient for accurate calibration of the sensitivity of a gamma camera. It is necessary to use the same collimator and to set the same energy windows like the ones that will be used in the acquisition protocols of the examined patients. In a work from 2017, Matsumoto et al. [20] confirmed that the quantitative calibration of the gamma camera made based on a planar sensitivity measurement (using a Petri dish) is burdened with a lower measurement error than the calibration based on the measurement and reconstruction of a homogeneous cylindrical phantom (CCF method).

Even though a correct cross-calibration is performed, some differences in quantitative measurements might occur between centres because of the differences in applied image reconstruction parameters. Reconstructions should be performed with corrections for attenuation, scatter and resolution recovery. Therefore, it is necessary to standardize the method of image reconstruction for CCF and then to standardize the technique of quantitative SPECT/CT systems calibration.

The study performed by Gnesin et al. [2] shows that relative lung errors were comparable to PET levels, which suggest the efficient integration of attenuation and scatter corrections. Its value was less than $10 \%$ in the case of background activity concentrations $>12 \mathrm{kBq} / \mathrm{mL}$. However, the value increased with lower statistic and was found to be approximately $20 \%$ for background activity concentrations of $1.5 \mathrm{kBq} / \mathrm{mL}$.

Recovery coefficient (or Accuracy) is a very helpful quantity to assess the effectiveness of SPECT/CT quantitative analysis. By plotting RC curves for phantom hot spheres of various sizes, the optimal quantitative image reconstruction technique can be easily established. A phantom containing hot spheres is required to measure RC, Accuracy and contrast recovery measurements. A cylindrical phantom filled with a homogeneous radioisotope solution is sufficient to determine the background calibration factor, total activity and image noise. Relative error measurement requires the use of an anthropomorphic phantom with a lung insert.

The accuracy of the quantitative SPECT/CT technique has been evaluated with phantom experiments in many works. The results show that it is possible to obtain reproducible measurements. The study performed by Peters et al. [11] shows that absolute SPECT quantification is achievable in a multi-centre and multi-vendor setting. The variation between centres is mainly caused by the use of different reconstruction algorithms and/or settings. Based on their research, the authors confirmed that the OSEM iterative method with 5 iterations and 15 subsets, without the
3D post-reconstruction filter, is the optimal clinical method for the reconstruction of quantitative images obtained with the NM/CT850 gamma camera $[21,12]$. On the other hand, for the gamma camera Symbia Intevo, Gnesin et al. [2] chose the iterative reconstruction technique with 16 iterations and 4 subsets and Gaussian smoothing of FWHM $7.5 \mathrm{~mm}$ (for foci with a diameter greater than $24 \mathrm{~mm}$ ) as the optimal method.

The next step should be to establish the equivalence between the corresponding reconstruction techniques for gamma cameras from different manufacturers, for analogous acquisition parameters. The aim of the work should be focused on finding equivalent techniques of image reconstruction and filtering, which should ensure the same level of accuracy in the measurements of activity concentration for different SPECT/CT systems.

The further aim should be to unify and standardize calibration and quality control procedures for all types of new QSPECT/CT systems.

\section{Summary}

Quantitative SPECT/CT requires proper calibration, optimization and quality control. Various types of modern SPECT/CT scanners have different software for calibration and quality control of imaging. There is still no standardization in this regard for quantitative SPECT/CT. This paper presents three selected physical quantities characterizing the quality of quantitative SPECT/CT system: sensitivity, SPECT Reconstructed Spatial Resolution and dead-time. The measurement of these parameters requires the use of standard gamma camera software for image acquisition and, also, external programs for quantitative analysis of recorded data. Seven values characterizing the quality of quantitative SPECT/CT images were also presented: background calibration factor, recovery coefficient, the accuracy of measurements, coefficient of variation for image noise evaluating, total activity deviation, contrast recovery and residual error in scatter and attenuation corrections. All seven parameters can be determined using only clinical software.

The authors believe that the standardization of the quantitative SPECT/CT technique could be significantly accelerated by the unification of quantitative acquisition techniques, reconstruction, image analysis, as well as quality control procedures in all commercial quantitative SPECT/CT systems.

\section{References}

1. NEMA Standards Publication NU 2-2018. Performance measurements of positron emission tomographs (PETS) Rosslyn, 2018.

2. Gnesin S, Leite Ferreira P, Malterre J, et al. Phantom validation of Tc-99m absolute quantification in a SPECT/CT commercial device. Comput Math Methods Med. 2016; 2016: 4360371, doi: 10.1155/2016/4360371, indexed in Pubmed: 28096891.

3. Ferrando O, Chimenz A, Foppiano F, et al. SPECT/CT activity quantification in 99mTc-MAA acquisitions. J Diag Imaging Ther. 2018; 5(1): 32-36, doi: 10.17229/jdit.2018-0624-034.

4. Ryu $\mathrm{H}$, Meikle SR, Willowson KP, et al. Performance evaluation of quantitative SPECT/CT using NEMA NU 2 PET methodology. Phys Med Biol. 2019; 64(14): 145017, doi: 10.1088/1361-6560/ab2a22, indexed in Pubmed: 31207583.

5. Dickson J, Ross J, Vöö S. Quantitative SPECT: the time is now. EJNMMI Phys. 2019; 6(1): 4, doi: 10.1186/s40658-019-0241-3, indexed in Pubmed: 30830530 . 
6. Ogawa K, Harata Y, Ichihara T, et al. A practical method for position-dependent Compton-scatter correction in single photon emission CT. IEEE Trans Med Imaging. 1991; 10(3): 408-412, doi: 10.1109/42.97591, indexed in Pubmed: 18222843.

7. NEMA Standards Publication NU 1-2018. Performance measurements of gamma cameras Rosslyn, 2018.

8. QDOSE Dosimetry Software. User Manual. Version 1.1.14. 2020.

9. Cranley K, Millar R, Bell TK. Correction for deadtime losses in a gamma camera/data analysis system. Eur J Nucl Med. 1980; 5(4): 377-382, doi: 10.1007/BF00445626, indexed in Pubmed: 7398673.

10. Boellaard R, O'Doherty MJ, Weber WA, et al. FDG PET and PET/CT: EANM procedure guidelines for tumour PET imaging: version 1.0. Eur J Nucl Med Mol Imaging. 2010; 37(1): 181-200, doi: 10.1007/s00259-009-1297-4, indexed in Pubmed: 19915839.

11. Peters SMB, van der Werf NR, Segbers M, et al. Towards standardization of absolute SPECT/CT quantification: a multi-center and multi-vendor phantom study. EJNMMI Phys. 2019; 6(1): 29, doi: 10.1186/s40658-019-0268-5, indexed in Pubmed: 31879813.

12. NM Quantification Q.Metrix for SPECT/CT Package. White Paper DOC1951185: GE Healthcare.

13. Koopman D, van Osch JAC, Jager PL, et al. Technical note: how to determine the FDG activity for tumour PET imaging that satisfies European guidelines. EJNMMI Phys. 2016; 3(1): 22, doi: 10.1186/s40658-016-0158-z, indexed in Pubmed: 27682837.

14. Seret A, Nguyen D, Bernard C. Quantitative capabilities of four state-of-theart SPECT-CT cameras. EJNMMI Res. 2012; 2(1): 45, doi: 10.1186/2191219X-2-45, indexed in Pubmed: 22925467.
15. Zeintl J, Vija AH, Yahil A, et al. Quantitative accuracy of clinical 99mTc SPECT/CT using ordered-subset expectation maximization with 3-dimensional resolution recovery, attenuation, and scatter correction. J Nucl Med. 2010; 51(6): 921-928, doi: 10.2967/jnumed.109.071571, indexed in Pubmed: 20484423.

16. Ferrer L, Delpon $G$, Lisbona A, et al. Dosimetric impact of correcting count losses due to deadtime in clinical radioimmunotherapy trials involving iodine-131 scintigraphy. Cancer Biother Radiopharm. 2003; 18(1): 117-124 doi: 10.1089/108497803321269395, indexed in Pubmed: 12667314.

17. Beauregard JM, Hofman MS, Pereira JM, et al. Quantitative (177)Lu SPECT (QSPECT) imaging using a commercially available SPECT/CT system. Cancer Imaging. 2011; 11(1): 56-66, doi: 10.1102/1470-7330.2011.0012, indexed in Pubmed: 21684829.

18. Celler A, Piwowarska-Bilska H, Shcherbinin S, et al. Evaluation of dead-time corrections for post-radionuclide-therapy (177)Lu quantitative imaging with low-energy high-resolution collimators. Nucl Med Commun. 2014; 35(1): 7387, doi: 10.1097/MNM.0000000000000011, indexed in Pubmed: 24131941.

19. Dewaraja YK, Frey EC, Sgouros G, et al. MIRD pamphlet No. 23: quantitative SPECT for patient-specific 3-dimensional dosimetry in internal radionuclide therapy. J Nucl Med. 2012; 53(8): 1310-1325, doi: 10.2967/jnumed.111.100123, indexed in Pubmed: 22743252.

20. Matsutomo N, Matsumoto S, Yamamoto T, et al. Validation of a calibration method using the cross-calibration factor and system planar sensitivity in quantitative single-photon emission computed tomography imaging. Radiol Phys Technol. 2017; 10(4): 439-445, doi: 10.1007/s12194-017-0416-3, indexed in Pubmed: 28822095.

21. Piwowarska-Bilska $H$, Supińska A, Birkenfeld B. What validation tests can be done by the clinical medical physicist while waiting for the standardization of quantitative SPECT/CT imaging? , doi: 10.21203/rs.3.rs-118913/v1. 